\title{
Retracted: Comparative Functional Studies on Two Diploid Cotton Genomes Reveals Functional Differences of Basic Helix-loop-helix Proteins in Arabidopsis Trichome Initiation
}

\begin{abstract}
Anh Phu Nam Bui ${ }^{1,2}$, Vimal Kumar Balasubramanian ${ }^{3}$, Thuan-Anh Nguyen-Huu ${ }^{4}$, Tuan-Loc Le ${ }^{4}$ and Hoang Dung Tran ${ }^{4 *}$

${ }^{1}$ Faculty of Biotechnology, Ho Chi Minh city Open University, 35 Ho Hao Hon street, Ho Chi Minh city, Vietnam.

${ }^{2}$ Department of Cell Biology, Texas Tech University Health Sciences Center, 36014 th street, Lubbock, Texas 79430, USA.

${ }^{3}$ Pacific Northwest National Laboratory, 902 Battelle Blvd, Richland, WA 99354, USA.

${ }^{4}$ Faculty of Biotechnology, Nguyen Tat Thanh University, 298A-300A Nguyen Tat Thanh Street, Ward 13, District 4, Ho Chi Minh city 72820, Vietnam.

This work was carried out in collaboration among all authors. Author APNB designed the study, performed the statistical analysis, wrote the protocol and wrote the first draft of the manuscript. Authors VKB, TANH and TLL managed the analyses of the study. Author HDT supervised and edited the final manuscript. All authors read and approved the final manuscript.

Article Information

DOI: 10.9734/ARRB/2019/v34i230146 Editor(s):

(1) Dr. Tunira Bhadauria, Professor, Department of Zoology, Feroze Gandhi Post Graduate College, Kanpur University, U.P, Reviewers:

(1) Michael Bordonaro, Geisinger Commonwealth School of Medicine, USA. (2) Heba Gamal Abd El-Aziz Nasr, Al-Azhar University, Egypt. Complete Peer review History: http://www.sdiarticle4.com/review-history/53568

Original Research Article

Received 29 October 2019

Accepted 02 January 2020

Published 10 January 2020
\end{abstract}

\section{ABSTRACT}

Background: The cultivated tetraploid cotton species (AD genomes) was originated from two ancestral diploid species (A and D genomes). While the ancestral A-genome species produce spinnable fibers, the D- genome species do not. Cotton fibers are unicellular trichomes originating from seed coat epidermal cells, and currently there is an immense interest in understanding the 
process of fiber initiation and development. Current knowledge demonstrates that there is a great of deal of resemblance in initiation mechanism between by Arabidopsis trichome and cotton fiber.

Methodology: In this study, we performed comparative functional studies between A genome and D-genome species in cotton by using Arabidopsis trichome initiation as a model. Four cotton genes TTG3, MYB2, DEL61 and DEL65 were amplified from A-genome and D-genome species, and transformed into their homolog trichomeless mutants Arabidopsis $t t g 1, g / 1$, and $g / 3 e g / 3$, respectively.

Results: Our data indicated that the transgenic plants expressing TTG3 and MYB2 genes from Agenome and D-genome species complement the ttg1 and g/1 mutants, respectively. We also discovered complete absences of two functional basic helix loop helix (bHLH) proteins (DEL65/DEL61) in D- diploid species and one (DEL65) that is functional in A-genome species, but not from D-genome species. This observation is consistent with the natural phenomenon of spinnable fiber production in A-genome species and absence in D-genome species.

Keywords: Arabidopsis; trichome; MYB2; TTG3; DEL65; DEL61; A genome, D genomes,

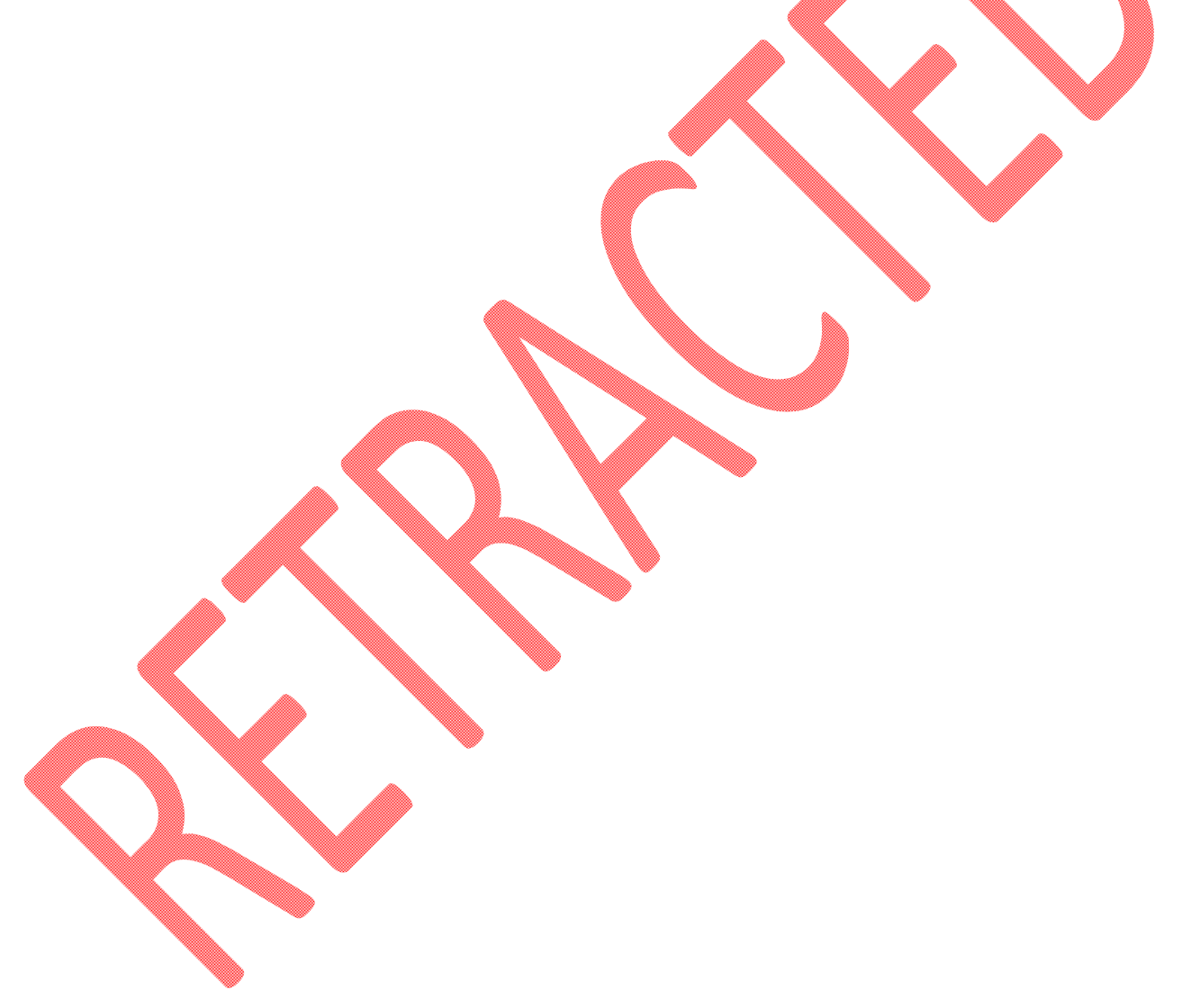

